\title{
A Framework for Building Design Management in an Imperfect BIM Environment
}

\author{
A. T. Kassem ${ }^{1}$ \\ ${ }^{1}$ Beni-suef University, Beni-suef, Egypt \\ Correspondence: A. T. Kassem, Faculty of Engineering, Beni-suef University, Beni-suef, Egypt. E-mail: \\ Abdelraouf_kassem@yahoo.com
}

Received: October 16, 2017

Accepted: November 20, 2017 Online Published: November 23, 2017

doi:10.5539/emr.v7n1p1

URL: http://doi.org/10.5539/emr.v7n1p1

\begin{abstract}
Building design process is a wide spectrum activity, regarding its complexity. It begins by simple compartments structures till large multistory administrative buildings, involving various specialties. Complex design management, where a multidisciplinary team or teams are involved in the design process; requires high level of collaboration to reach the optimized client goals economically and on time. BIM provides a wide range support for such an activity, where the most critical criterion in design management is ensuring the appropriate flow of information between design partners. Each participant in the design process should receive relevant data or information in a complete form and on time. And consequently design participant should send and share their design outputs with relevant personnel. The perfect BIM environment requires that all participants within the design process are using BIM supporting software, and vice versa. Markets where BIM application is still in the growing phase some of the design participants adopt BIM while others don't. The main target of this paper is to propose a collaborative scheme to ensure information flow between design participants, using COBIE forms and XBIM toolkit; in the case where some of the participants in design process are not using BIM supporting software.
\end{abstract}

Keywords: BIM, COBie, XBIM Xplorer, design management

\section{Introduction}

Mostly in environments, where BIM culture is still growing some of the participants in the design process adopt BIM based software, as a platform for their work; while others don't. This situation handicaps both BIM based and non BIM based participants, due to lack of reliable information transfer schemes between the two groups of participants. This situation has arisen the need for a tool oriented to bridging the gap between BIM using and non BIM using design participants. This paper presents such a tool in the form of a frame work, defining main design process elements, associated with two supporting tools. COBIE has been used for information transfer, while the BIM toolkit for information verification.

\section{COBie Forms}

COBie had been early invented to transfer data from the design to the construction to the facility management processes. They consist of a number of fixed format spread sheets, summarizing projects information. Each COBie form deals with specific type of information. This paper presents COBie forms not only to fill in the missing node in data transfer chain between design, construction, and facility management; but also as a tool for bridging the gap between BIM and non-BIM users. COBie forms have been automatically generated using the COBie extension plugin, as shown in Figure 1. It is not a must to transfer all COBie sheets every exporting cycle. A selective approach is to be adopted, to decide which sheets are to be exported, according to data required for sharing. 


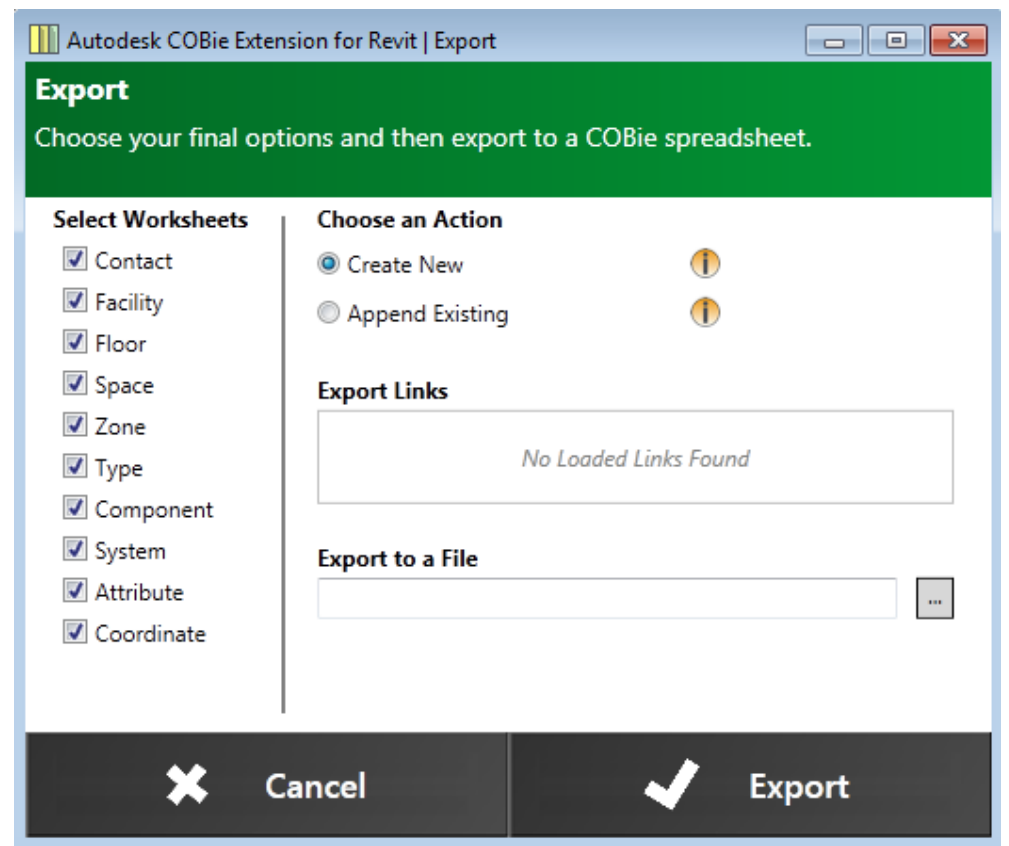

Figure 1. COBie extension plugin for revit

\section{XBIM Xplorer}

The XBIM Toolkit is an open source tool established to allow opening and rendering BIM models, in addition checking building model contents, to ensure all required fields are dragged into the model, defining various element's properties. It is compatible with IFC format and consequently able to support all software packages, prepared in accordance with IFC.XBIM Xplorer can't judge the quality of the BIM model contents. It just states whether the required fields are filled or empty. This nature of IFC clarifies its role in the imperfect BIM environment, where it allows human role to arise in managing to complete the BIM model. Figure 2 shows the XBIM Xplorer user interface, where information is represented in tabular and graphical forms.

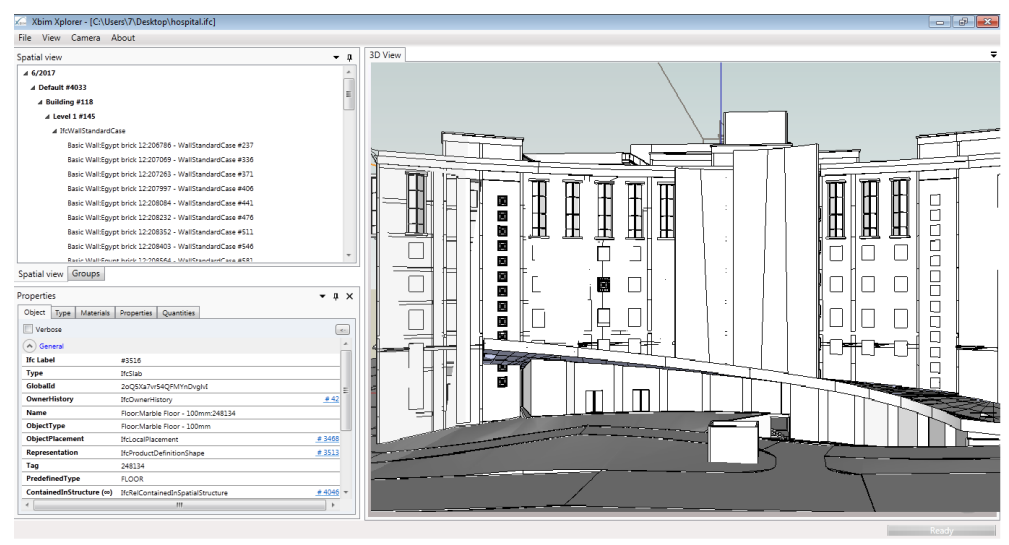

Figure 2. XBIM Xplorer user interface

\section{Building Information History}

Figure 3 shows patterns of building information history in case of BIM, Imperfect BIM, and non-BIM approaches. The perfect BIM environment ensures smooth flow of information throughout the design and construction processes. Once construction finishes a drop in processed data takes place, due to the use of COBie forms that keeps only essential information, required for facility management. On the other hand the non-BIM 
based approach faces multiple data drops between different stages throughout the building life cycle. The imperfect BIM environment design represents a compromise between the perfect BIM and the non-BIM approaches. The imperfect BIM environment approach sustains a smooth flow of data between various stages along building life cycle, in the same pattern as perfect BIM approach; but conserving a lower amount of building information, because only some of the design parties are using BIM tools.

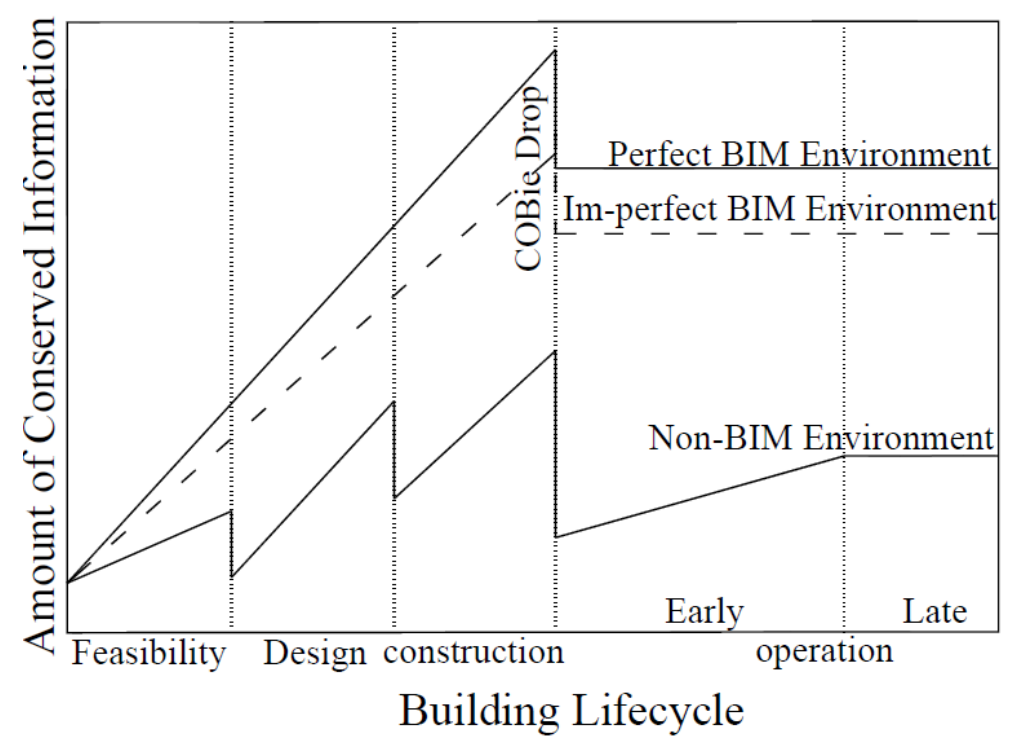

Figure 3. Building conserved information history

\section{The Proposed Framework}

The COBie forms in conjunction with XBIM Xplorerare considered complementary tools, used to ensure that the general BIM model is functioning efficiently. XBIM Xplorer is directly used in a frequent bases during the design to tune the process and validate the adequacy of model embedded data. While COBie is used for both documentation in a text form and assisting non-BIM participants to proceed in design activities.

Research body has been formulated as a flowchart, by which the implementation process is clarified. Each step in the flow chart is clearly formulated and inter-relationships between various steps are also presented. Figure 4 shows successive steps of the framework in the form of a flowchart.

\section{Framework Implementation}

Implementation of the proposed framework has been performed in a redesign process of a hospital project. The redesign was a client requirement to take into account some modifications, required for equipment installation. These modifications required re-assigning of spaces within different floors. Figures 5 to 16 show the reassigned floor spaces, in addition to isometrics and inter-plans relationships. Tables 1 up to 3 show data associated with the functional plans, in the form of COBie spread sheets; to complete the image for reassigned spaces for non-BIM participants.

A general goal throughout the design process, is to ensure all data required for each design activity are available to the responsible in a suitable form and on time. Some data may require verification, others need acceptance, while other data should be avoided. Disciplinary protocols should define for each discipline what type of data must be verified, or should be accepted, or could be avoided. 


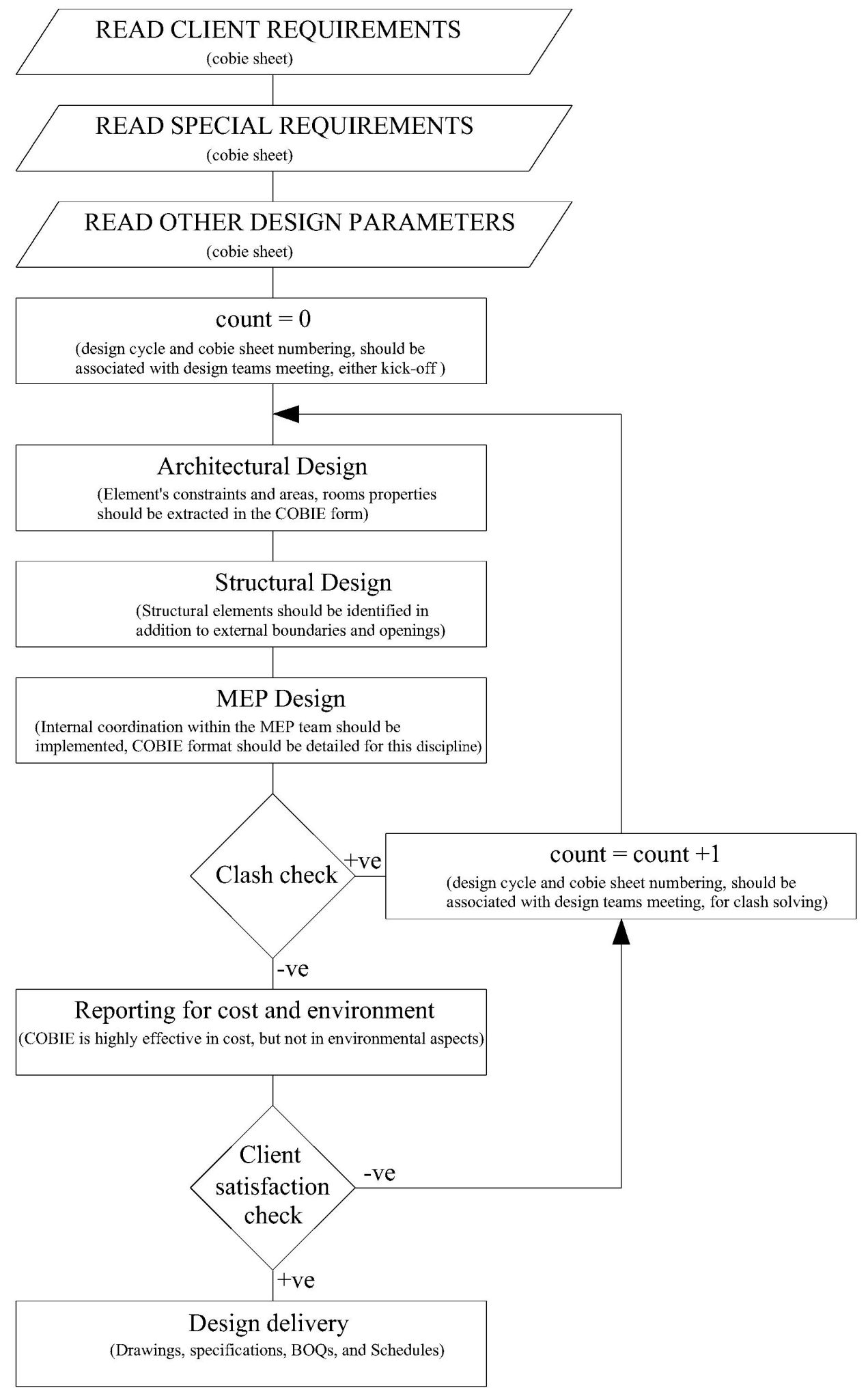

Figure 4. Proposed framework flowchart 


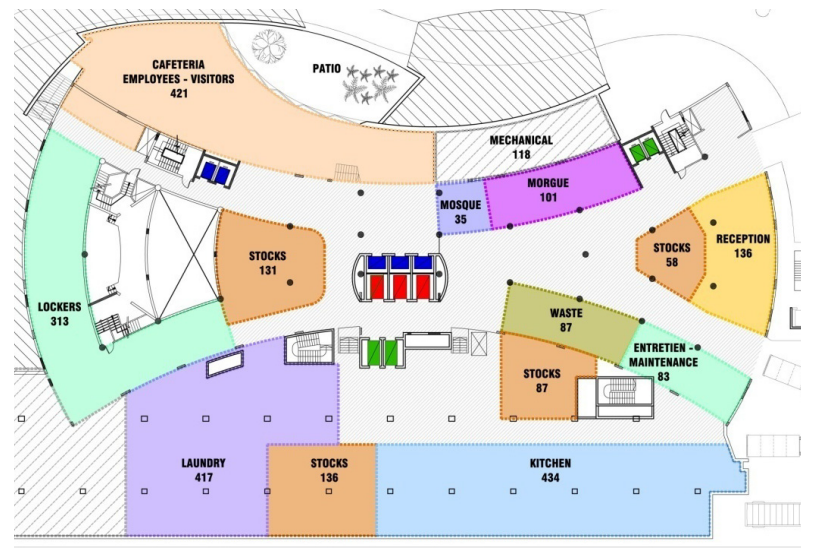

Figure 5. Level [-2] Functional Plan

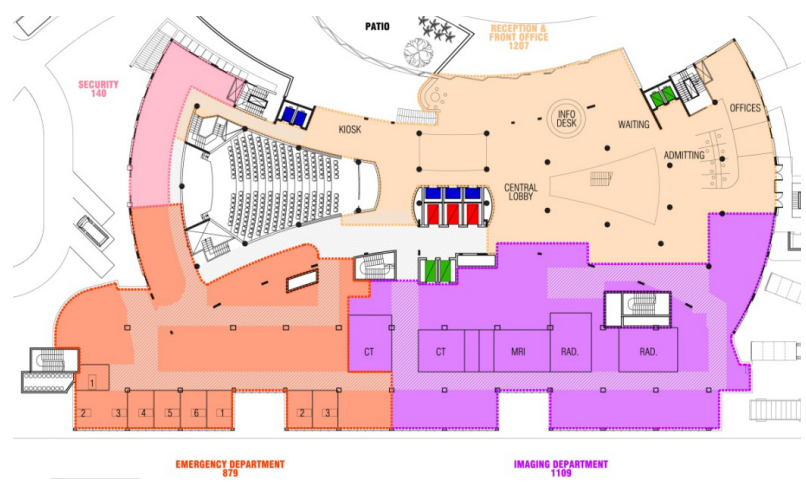

Figure 6. Level [-1] Functional Plan

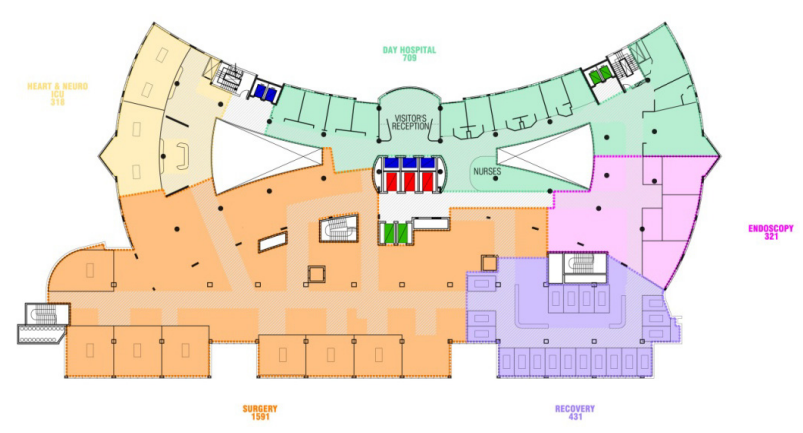

Figure 9. Level [02] Functional Plan

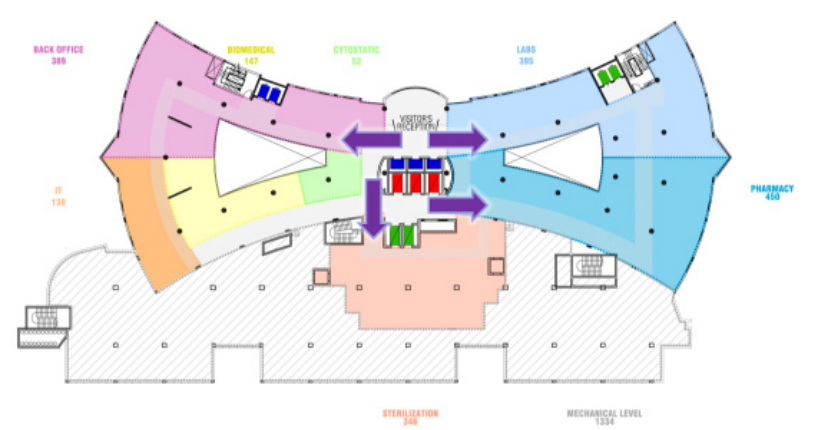

Figure 10. Level [03] Functional Plan

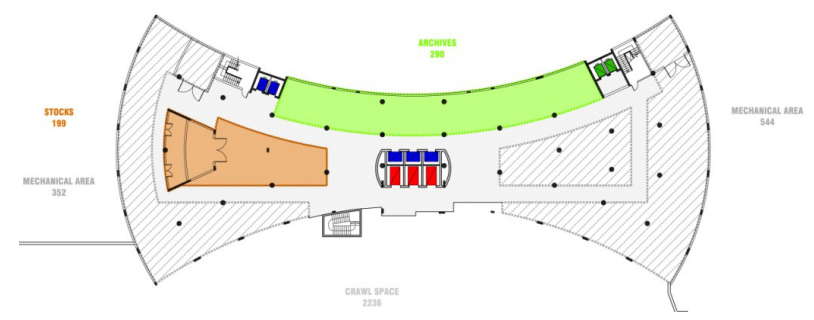

Figure 7. Level 00] Functional Plan

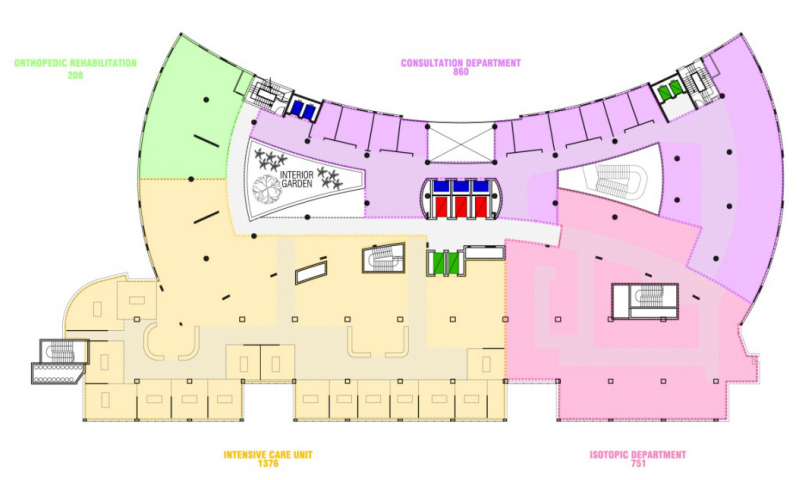

Figure 8. Level [01] Functional Plan

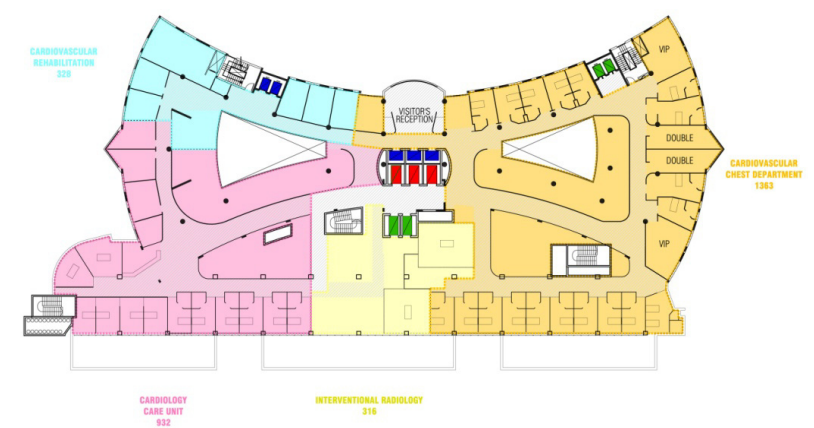

Figure 11. Level [04] Functional Plan

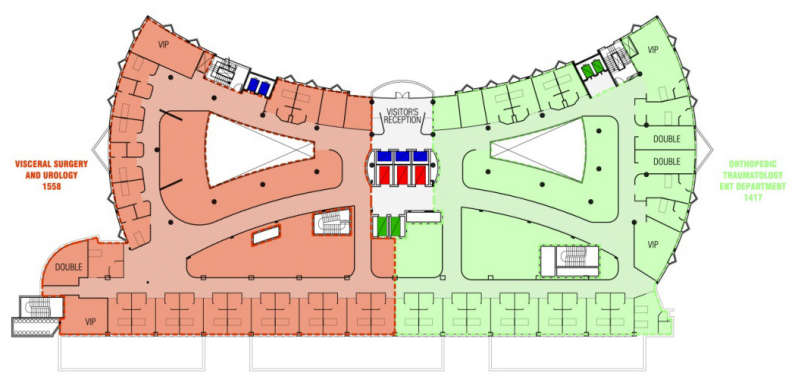

Figure 12. Level [05] Functional Plan 


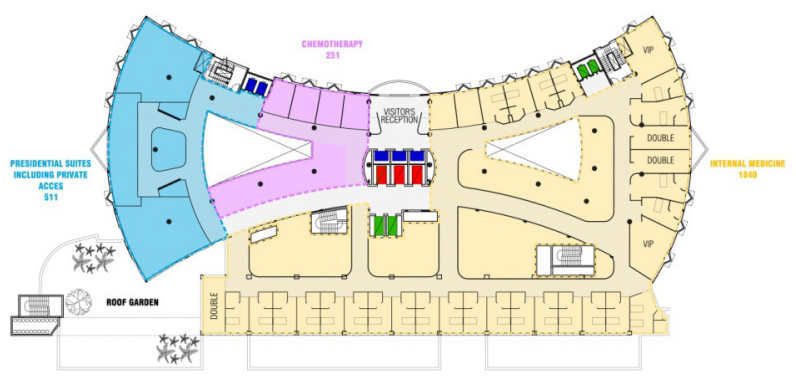

Figure 13. Level [06] Functional Plan

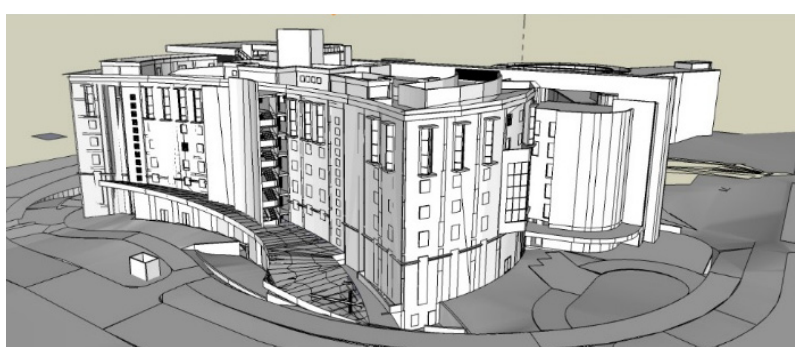

Figure 14. North-west Isometric

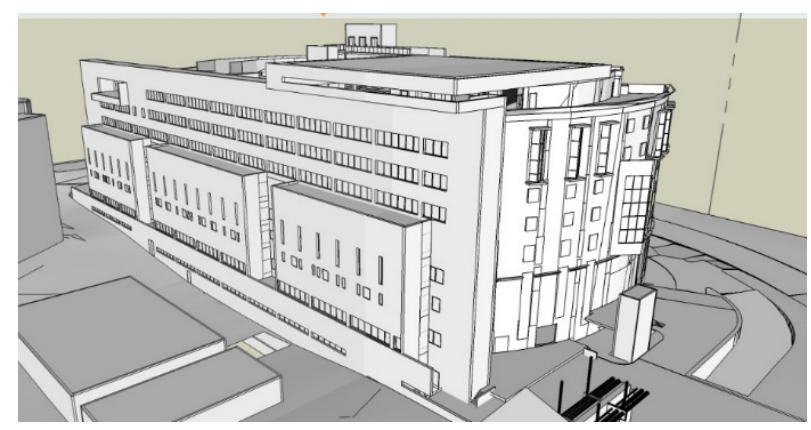

Figure 15. South-East Isometric

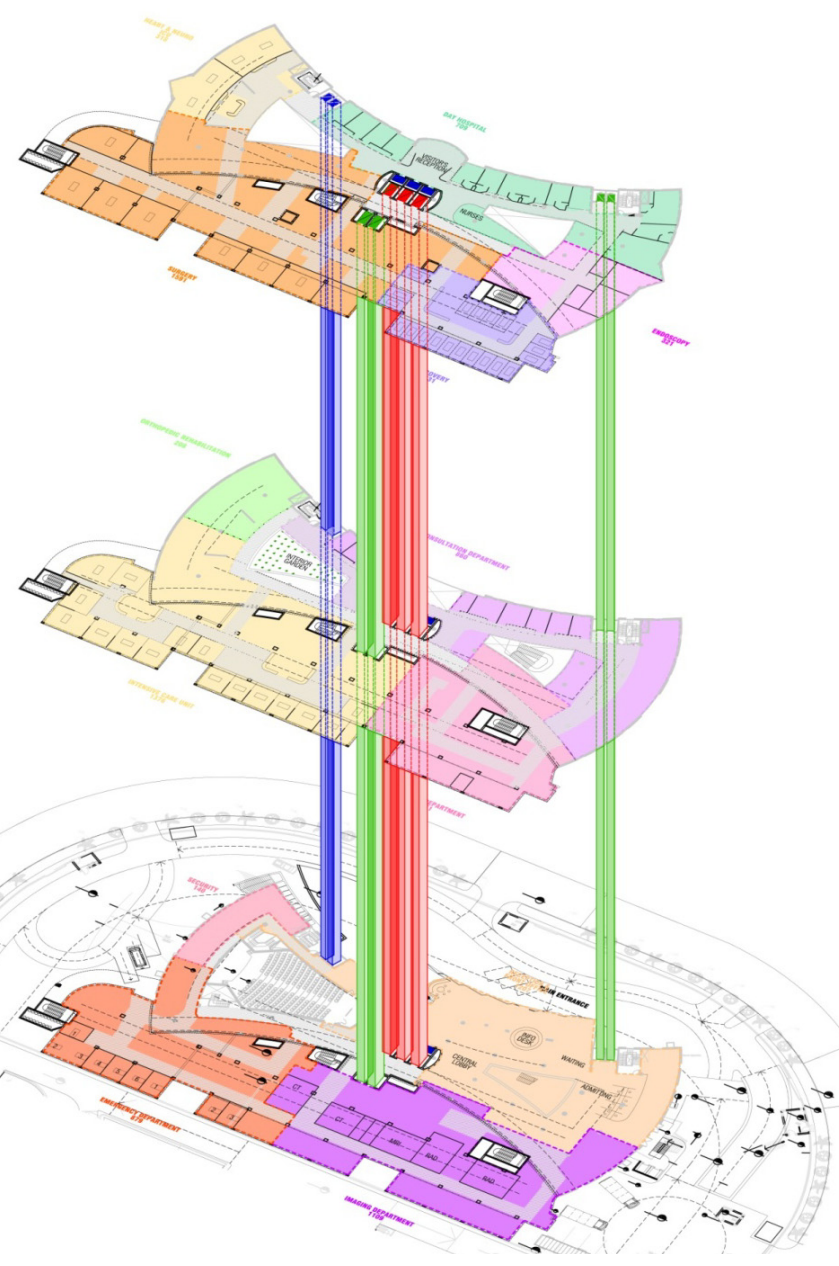

Figure 16. Multiple Plans Relations

Table 1. Contact spread sheet

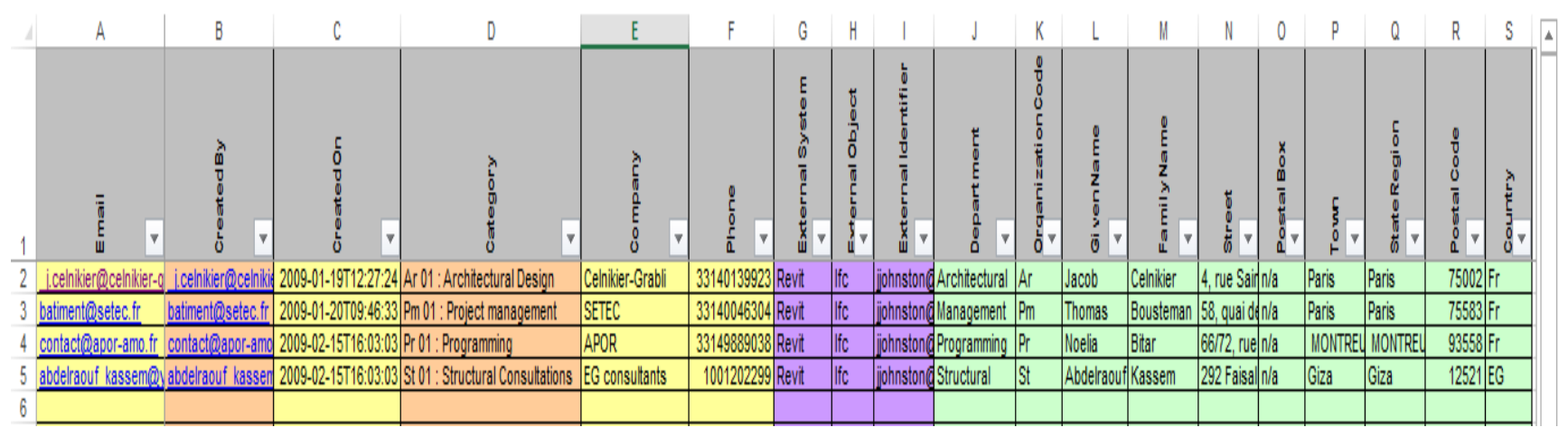


Table 2. Floor spread sheet

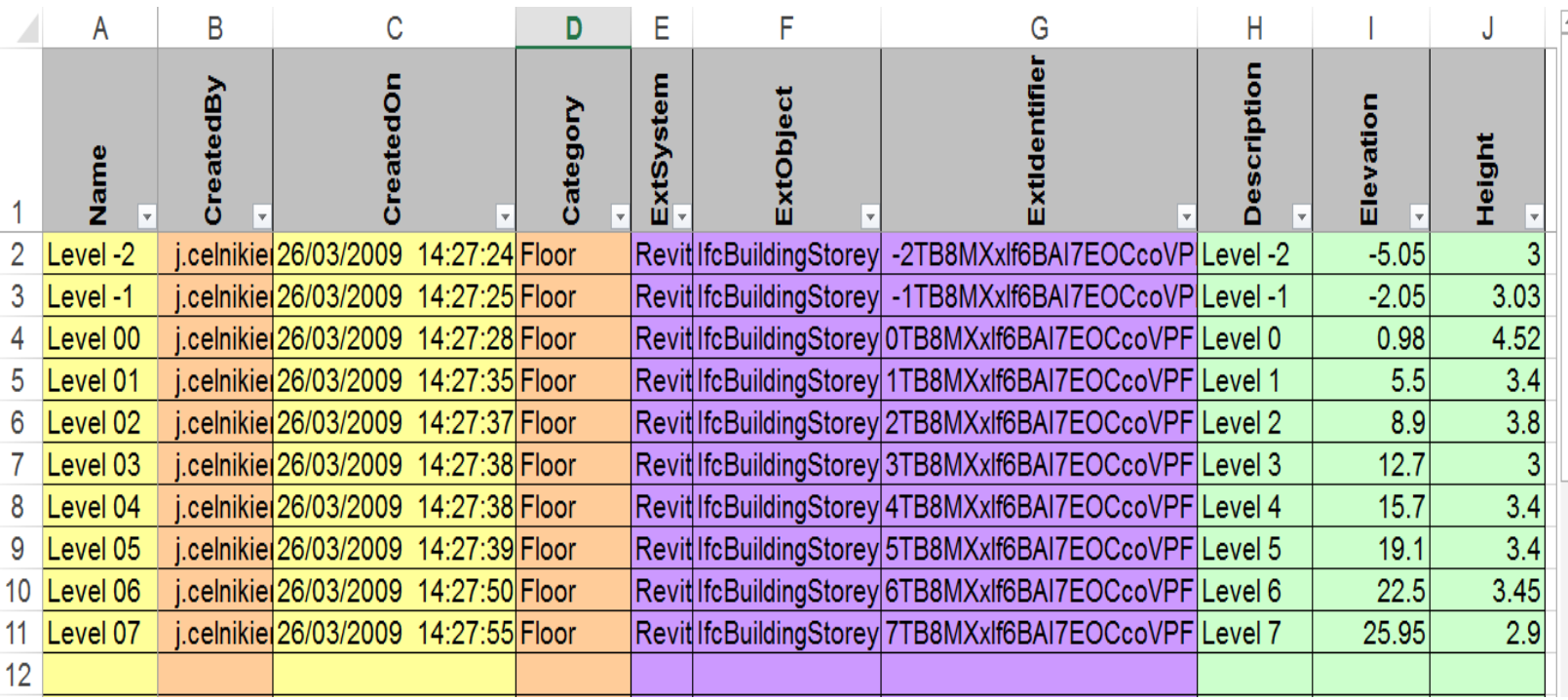

Table 3. Space spread sheet

\begin{tabular}{|c|c|c|c|}
\hline FloorName & Description & ExtSystem & ExtObject \\
\hline Level 0 & N.A. & Revit & IfcSpace \\
\hline Level 0 & N.A. & Revit & IfcSpace \\
\hline Level 0 & N.A. & Revit & IfcSpace \\
\hline Level -1 & N.A. & Revit & IfcSpace \\
\hline Level 0 & N.A. & Revit & IfcSpace \\
\hline Level 0 & N.A. & Revit & IfcSpace \\
\hline Level 1 & N.A. & Revit & IfcSpace \\
\hline Level 2 & N.A. & Revit & IfcSpace \\
\hline Level 4 & N.A. & Revit & IfcSpace \\
\hline Level 1 & N.A. & Revit & IfcSpace \\
\hline Level 1 & N.A. & Revit & IfcSpace \\
\hline Level 0 & N.A. & Revit & IfcSpace \\
\hline Level 0 & N.A. & Revit & IfcSpace \\
\hline Level 0 & N.A. & Revit & IfcSpace \\
\hline Level 0 & N.A. & Revit & IfcSpace \\
\hline Level 0 & N.A. & Revit & IfcSpace \\
\hline Level 0 & N.A. & Revit & IfcSpace \\
\hline Level 1 & N.A. & Revit & IfcSpace \\
\hline Level 2 & N.A. & Revit & IfcSpace \\
\hline Level 2 & N.A. & Revit & IfcSpace \\
\hline Level 4 & N.A. & Revit & IfcSpace \\
\hline Level 2 & N.A. & Revit & IfcSpace \\
\hline Level 1 & N.A. & Revit & IfcSpace \\
\hline Level 4 & N.A. & Revit & IfcSpace \\
\hline Level 2 & N.A. & Revit & IfcSpace \\
\hline
\end{tabular}

ExtIdentifier

RoomTag

UsableHeight

GrossArea NetArea

Front office (Admitting / Billng department)

Security department

Customer service - patients account

Customer service - patients accoun

Conferences /meeting room

Out patients clinics

Day hospit. Dep

Cardiovascular Rehabilitation

Orthopedic Rehabilitation

Day hospit. Dept

Adults Emergency (20 000/year )

Radiology and MRI/CT's receptio

Radiology / echography and mammography are

MRI / CT - Programmed patients

$\mathrm{CT} /$ emergencies and multislice coronary

Utility for radiology and MRI/CT

Nuclear medicine (scientigraphy + Pet Scan)

Operating rooms

Recovery area

Cardiology and interventional radiology

Endoscopy Department

ICU

Heart and Neuro ICU

$\begin{array}{lcccc}\text { N.A. } & \text { A1 } & 7 & 154 & 154 \\ \text { N.A. } & \text { A2 } & 7 & 111 & 111 \\ \text { N.A. } & \text { A3 } & 3.2 & 39 & 39 \\ \text { N.A. } & \text { A4.1 } & 3.2 & 85 & 85 \\ \text { N.A. } & \text { A4.2 } & 3.2 & 40 & 40 \\ \text { N.A. } & \text { A5 } & 7 & 800 & 800 \\ \text { N.A. } & \text { B1 } & 3.2 & 559 & 559 \\ \text { N.A. } & \text { B2 } & 3.2 & 475 & 475 \\ \text { N.A. } & \text { B3 } & 3.2 & 170 & 170\end{array}$

N.A. B3

N.A. B

N.A. B5

N.A. $\quad$ Cl

N.A. D

N.A. D2

N.A. D3

N.A. D4

N.A. D

N.A. $\quad$ D6

N.A. $\quad$ E1

N.A. E2

N.A. E4

N.A.

F1

F2
N.A. E3 


\begin{tabular}{|c|c|c|c|}
\hline j.celnikier@celnikier-grabli.com & $22 / 04 / 2009$ & 13:10:11 PM & Hospitalization level \\
\hline CreatedBy & \multicolumn{2}{|c|}{ CreatedOn } & Category \\
\hline j.celnikier@celnikier-grabli.com & $15 / 04 / 2009$ & 13:14:52 PM & Hospitalization level \\
\hline j.celnikier@celnikier-grabli.com & 2009-04-1 & 4T00:43:44 & Hospitalization level \\
\hline j.celnikier@celnikier-grabli.com & 2009-04-1 & 4T10:11:22 & Pharmacy \\
\hline j.celnikier@celnikier-grabli.com & 2009-04-2 & $8 \mathrm{~T} 10: 12: 44$ & Cytostatic production area \\
\hline j.celnikier@celnikier-grabli.com & 2009-04-1 & 3T12:27:24 & Sterilization \\
\hline j.celnikier@celnikier-grabli.com & 2009-04-2 & $1 \mathrm{~T} 11: 17: 11$ & Laboratories \\
\hline j.celnikier@celnikier-grabli.com & 29/04/2009 & 16:12:35 PM & Mortuary \\
\hline j.celnikier@celnikier-grabli.com & $22 / 04 / 2009$ & 13:10:11 PM & Biomedical Maintenance workshop \\
\hline j.celnikier@celnikier-grabli.com & 2009-04-2 & $8 \mathrm{~T} 10: 12: 44$ & Administriv archive \\
\hline j.celnikier@celnikier-grabli.com & 29/04/2009 & 16:12:35 PM & Administriv and medical archive of the patient \\
\hline j.celnikier@celnikier-grabli.com & $15 / 04 / 2009$ & 13:14:52 PM & IT (Information Technology) service \\
\hline j.celnikier@celnikier-grabli.com & 2009-04-2 & $1 \mathrm{~T} 11: 17: 11$ & Head Dpt \\
\hline j.celnikier@celnikier-grabli.com & 2009-04-1 & 4T00:43:44 & Human ressources Dpt \\
\hline j.celnikier@celnikier-grabli.com & $16 / 04 / 2009$ & 16:36:14 PM & Direction \\
\hline j.celnikier@celnikier-grabli.com & 2009-04-1 & 4T10:11:22 & Employees locker room \\
\hline j.celnikier@celnikier-grabli.com & $29 / 04 / 2009$ & 16:12:35 PM & Employees and visitors cafeteria \\
\hline j.celnikier@celnikier-grabli.com & $15 / 04 / 2009$ & 13:14:52 PM & Guarding medical \\
\hline j.celnikier@celnikier-grabli.com & 22/04/2009 & 13:10:11 PM & Reception / shipping \\
\hline j.celnikier@celnikier-grabli.com & 2009-04-2 & $8 \mathrm{~T} 10: 12: 44$ & Stocks \\
\hline j.celnikier@celnikier-grabli.com & $16 / 04 / 2009$ & 16:36:14 PM & Kitchen \\
\hline j.celnikier@celnikier-grabli.com & 2009-04-2 & $1 \mathrm{~T} 11: 17: 11$ & Waste room (infectious risk) \\
\hline j.celnikier@celnikier-grabli.com & $15 / 04 / 2009$ & 13:14:52 PM & Premises' hygiene \\
\hline j.celnikier@celnikier-grabli.com & 2009-04-1 & 3Т12:27:24 & Laundry room \\
\hline j.celnikier@celnikier-grabli.com & 5/04/2009 & 13:14:52 PI & Maintenan \\
\hline
\end{tabular}

\begin{tabular}{|c|c|c|c|c|c|c|c|c|}
\hline Level 4 & N.A. & Revit & IfcSpace & N.A. & G4 & 2.9 & 887 & 887 \\
\hline FloorName & Description & ExtSystem & ExtObject & ExtIdentifier & RoomTag & UsableHeight & GrossArea & NetAre: \\
\hline Level 5 & N.A. & Revit & IfcSpace & N.A. & G5 & 2.9 & 1829 & 1829 \\
\hline Level 6 & N.A. & Revit & IfcSpace & N.A. & G6 & 2.9 & 1445 & 1445 \\
\hline Level 3 & N.A. & Revit & IfcSpace & N.A. & $\mathrm{H} 1$ & 2.9 & 331 & 331 \\
\hline Level 3 & N.A. & Revit & IfcSpace & N.A. & $\mathrm{H} 2$ & 3.2 & 68 & 68 \\
\hline Level 3 & N.A. & Revit & IfcSpace & N.A. & $\mathrm{H} 3$ & 2.9 & 277 & 277 \\
\hline Level 3 & N.A. & Revit & IfcSpace & N.A. & $\mathrm{H} 4$ & 2.9 & 500 & 500 \\
\hline Level -1 & N.A. & Revit & IfcSpace & N.A. & H5 & 3.2 & 80 & 80 \\
\hline Level 3 & N.A. & Revit & IfcSpace & N.A. & H6 & 3.2 & 83 & 83 \\
\hline Level 0 & N.A. & Revit & IfcSpace & N.A. & I1 & 3.2 & 70 & 70 \\
\hline Level 0 & N.A. & Revit & IfcSpace & N.A. & I2 & 3.2 & 205 & 205 \\
\hline Level 3 & N.A. & Revit & IfcSpace & N.A. & 13 & 2.9 & 111 & 111 \\
\hline Level 3 & N.A. & Revit & IfcSpace & N.A. & J1.1 & 2.9 & 162 & 162 \\
\hline Level 4 & N.A. & Revit & IfcSpace & N.A. & $\mathrm{J} 1.2$ & 2.9 & 103 & 103 \\
\hline Level 6 & N.A. & Revit & IfcSpace & N.A. & $\mathrm{J} 1.3$ & 2.9 & 45 & 45 \\
\hline Level -1 & N.A. & Revit & IfcSpace & N.A. & K & 3.2 & 536 & 536 \\
\hline Level -1 & N.A. & Revit & IfcSpace & N.A. & K2 & 3.2 & 277 & 277 \\
\hline Level 3 & N.A. & Revit & IfcSpace & N.A. & K3 & 2.9 & 24 & 24 \\
\hline Level -1 & N.A. & Revit & IfcSpace & N.A. & L1 & 3.2 & 84 & 84 \\
\hline Level -1 & N.A. & Revit & IfcSpace & N.A. & L2 & 3.2 & 356 & 356 \\
\hline Level -1 & N.A. & Revit & IfcSpace & N.A. & L3 & 3.2 & 518 & 518 \\
\hline Level -1 & N.A. & Revit & IfcSpace & N.A. & L4) & 3.2 & 105 & 105 \\
\hline Level -1 & N.A. & Revit & IfcSpace & N.A. & L5 & 3.2 & 118 & 118 \\
\hline Level -1 & N.A. & Revit & IfcSpace & N.A. & L6 & 3.2 & 424 & 424 \\
\hline Level -1 & N.A. & Revit & IfcSpace & N.A. & L7 & 3.2 & 240 & 240 \\
\hline
\end{tabular}

\section{Conclusion}

1) The proposed framework proved its reliability in information sharing.

2) The framework minimized loss of data through conversion from a form to another during design and construction stage.

3) The framework decreased required time for design implementation, by efficiently sharing data and decreasing inquires.

4) COBie forms could be applied within the design stage, even before beginning of construction activities.

5) The proposed framework could bring BIM and non-BIM participants to the same platform.

6) The frame work could be implemented in environments, where BIM is still a new culture.

\section{References}

Abhijeet, D., Salman, A., \& Shreekanth, A. (2014). A framework for BIM-based knowledge management system. Creative construction conference 2014, CC 2014, Procedia Engineering, 85(2014), 113-122. https://doi.org/10.1016/j.proeng.2014.10.535

Aram, S. et al. (2013). Requirements for BIM platforms in the concrete reinforcement supply chain. Journal of Automation in Construction, 35(2013), 1-17. https://doi.org/10.1016/j.autcon.2013.01.013

BS 1192:2007+A1:2015, Collaborative production of architectural, engineering and construction information Code of practice 
Chen, K., Lu, W. S., Peng, Y., Steve, R., \& George, Q. H. (2015). Bridging BIM and building: From a literature review to an integrated conceptual framework. International Journal of Project Management, 33(2015), 1405-1416. https://doi.org/10.1016/j.ijproman.2015.03.006

Christoph, M., \& Bjorn, E. M. (2015). Effective digital collaboration in the construction industry-A case study of BIM deployment in a hospital construction project. Journal of Computers in Industry, 73(2015), 1-7. https://doi.org/10.1016/j.compind.2015.07.003

Hannes, L., \& Susanna, V. (2015). BIM implementation and organisational change: A case study of a large Swedish public client. 8th Nordic Conference on Construction Economics and Organization, Procedia Economics and Finance, 21(2015), 178-184. https://doi.org/10.1016/S2212-5671(15)00165-3

Hyunjoo, K., Zhenhua, S., Inhan, K., Karam, K., Annette, S., \& Jungho, Y. (2016). BIM IFC information mapping to building energy analysis (BEA) model with manually extended material information. Journal of Automation in Construction, 68(2016), 183-193. https://doi.org/10.1016/j.autcon.2016.04.002

McArthur, J. J. (2015). A Building Information Management (BIM) framework and supporting case study for existing building operations, maintenance and sustainability. International Conference on Sustainable Design, Engineering and Construction, Procedia Engineering, 118(2015), 1104-1111. https://doi.org/10.1016/j.proeng.2015.08.450

PAS 1192-2:2013, Incorporating Corrigendum No, Specification for information management for the capital/delivery phase of construction projects using building information modelling.

PAS 1192-3:2014, Incorporating Corrigendum No. 1, Specification for information management for the operational phase of assets using building information modelling.

PAS 1192-5:2015, Specification for security-minded building information modelling, digital built environments and smart asset management.

Väino, T. (2015). A BIM collaboration lab for improved through life support. 8th Nordic Conference on Construction Economics and Organization, Procedia Economics and Finance, 21(2015), 383-390. https://doi.org/10.1016/S2212-5671(15)00190-2

Yang, Z., Arto, K., \& Stephen, W. J. (2017). A review of risk management through BIM and BIM-related technologies. Journal of Safety Science, 97(2017), 88-98. https://doi.org/10.1016/j.ssci.2015.12.027

\section{Copyrights}

Copyright for this article is retained by the author(s), with first publication rights granted to the journal.

This is an open-access article distributed under the terms and conditions of the Creative Commons Attribution license (http://creativecommons.org/licenses/by/4.0/). 